\title{
Combined Treatment With Prednisone and Bromocriptine Does Not Influence the Pregnancy Outcome in Fresh Embryo Transfer Cycles: a Retrospective Study
}

\section{Tao Wang}

reproductive medicine center

Xin Mu

reproductive medicine center

Chun-rong Qin

reproductive medicine center

Yan Xia ( $\nabla$ airxia@126.com )

reproductive medicine center

Research

Keywords: Bromocriptine, prednisone, ovarian hyperstimulation syndrome, assisted reproductive techniques

Posted Date: December 11th, 2020

DOI: https://doi.org/10.21203/rs.3.rs-122467/v1

License: (c) (1) This work is licensed under a Creative Commons Attribution 4.0 International License.

Read Full License 


\section{Abstract}

Background: Ovarian hyperstimulation syndrome (OHSS) is a severe complication for infertile patients undergoing assisted reproduction technology (ART) treatment. Bromocriptine and prednisone are often used for treating OHSS. This study aimed to evaluate whether combined treatment with prednisone and bromocriptine can reduce the risk of OHSS and influence pregnancy outcomes in fresh embryo transfer cycles.

Methods: This retrospective study recruited infertile patients undergoing assisted reproduction techniques. We included 4,114 cycles of infertile patients who underwent IVF or ICSI treatment between January 2014 and May 2017 and divided them into two groups: the combined-treatment group and the single-treatment group.

Results: The OHSS cancellation rates in the combined- and single-treatment (control) groups were $31.50 \%$ and $40.88 \%$, respectively. There was a significant reduction in the OHSS cancellation rate between the combined- and single-treatment groups $(P<0.001)$. Single- and double-embryo transfers were completed in 350 and 495 cycles, respectively, in the combined-treatment group and in 227 and 517 cycles, respectively, in the single-treatment group $(P<0.001)$. The total number of successful embryo implantation was significantly different between the combined-treatment group (509 cycles, $40.88 \%$ ) and single-treatment groups (442 cycles, 38.30\%) $(P=0.012)$. There was no statistically significant difference in the clinical pregnancy $(P=0.492)$, miscarriage $(P=0.792)$, ongoing pregnancy $(P=0.719)$, live birth $(P=0.295)$, congenital abnormality $(P=0.081)$, and ectopic pregnancy occurrence rates $(P=0.649)$ between the combined- and single-treatment groups.

Conclusion: Compared with bromocriptine alone, combined treatment with bromocriptine and prednisone can better reduce the OHSS occurrence rate and does not affect the pregnancy outcomes in fresh embryo transfer cycles.

\section{Background}

Ovarian hyperstimulation syndrome (OHSS)[1] is a severe complication of controlled ovarian stimulation for infertile patients undergoing treatment using assisted reproduction technology (ART). It is characterized by the development of multiple follicles, enlarged ovaries, high estrogen levels, and increased capillary permeability [2]. It can also cause pleural ascites and blood concentration, liver, and kidney function abnormalities. Some complications such as thrombosis, multiple-organ failure, and even life-threatening complications that require hospitalization may occur in severe cases [3;4]. According to Sherwal et al.[5], the symptoms of mild OHSS included abdominal distension, discomfort, mild nausea/vomiting, and diarrhea. Moderate OHSS includes the symptoms of mild OHSS as well as ultrasonographic evidence of ascites (perpendicular fluid pocket $>9 \mathrm{~cm}^{2}$ ). Severe OHSS requires clinical evidence of ascites and/or hydrothorax and breathing difficulties or must satisfy one of the following criteria: increased blood viscosity (i.e., hematocrit level $\geq 45 \%$; leucocyte count $\geq 20,000 / \mathrm{mm}^{2}$ ), 
coagulation abnormality, diminished renal perfusion and function (serum creatinine $>1.2 \mathrm{mg} / \mathrm{dL}$ ), and/or liver dysfunction (defined as AST and/or ALT > $40 \mathrm{lU} / \mathrm{mL}$. Although many studies on OHSS prevention have been conducted, more effective measures for reducing the above-related symptoms in clinical practice are lacking. Therefore, exploring an effective prevention measure for OHSS has become a clinical research hotspot in ART treatment.

Prednisone is a class of intermediate-acting glucocorticoids that has a good effect on reducing the leakage of vasoactive substances and vascular permeability. As a common immunomodulatory agent, prednisone is often used for infertile patients with repeated embryo implantation failures [6-8] and for improvement of reproductive outcomes [9;10]. Prednisone also reduces exudation and eliminates edema in some inflammatory conditions or diseases. Tan et al.[11], Lainas et al.[12], and other studies[13] confirmed that prednisone had no side effect on in vitro fertilization (IVF) pregnancy rate. Moreover, compared with other preventive measures such as albumin, hydroxyethyl starch, and cycle cancellation, the use of oral prednisone for the prevention and treatment of OHSS had no risk of blood-borne diseases and reduced the financial burden of patients. Some studies showed that prednisone does not affect the IVF pregnancy rate, has minimal side effects [14], and has significant advantages in terms of economic burden and administration route.

Bromocriptine, a dopamine receptor agonist $[15 ; 5 ; 16]$, which inhibits vascular permeability by blocking vascular endothelial growth factor (VEGF)-receptor binding pathways, has been found to reduce angiogenic factor release and vascular permeability by inhibiting VEGF receptor phosphorylation [17-21]. Some studies have shown that vaginal administration of bromocriptine could reduce the probability of adverse reactions in the digestive tract and nervous system, and has a preventive effect on OHSS [16]. Hence, bromocriptine is often used for OHSS prevention [22]. Spitzer et al. [23] found that bromocriptine and cabergoline have similar OHSS preventive effects. A systematic review [24] showed that prophylactic treatment with a dopamine agonist can reduce the incidence, but not the severity, of OHSS without compromising pregnancy outcomes. Both cabergoline and bromocriptine can be administered to women with a high risk for OHSS by stimulating the dopamine D2 receptor [25;26;23]. In the early stage of acute inflammation, it can increase the tension of blood vessels, reduce congestion, and reduce capillary permeability. The release of inflammatory mediator reduces exudation and eliminates edema, which may have a preventive effect on OHSS. However, there is no study reporting the clinical application of combined use of bromocriptine and prednisone in IVF. Additionally, their long-term effect on pregnancy outcomes has not been confirmed. Therefore, this study aimed to compare these prophylactic treatments in preventing OHSS in women with a high risk of OHSS who undergo IVF or intracytoplasmic sperm injection (ICSI) and to evaluate the effects of the combination therapy on the quality of human embryos and patients' pregnancy outcomes.

\section{Methods}

\section{Design and setting}


This is a retrospective study was performed at the Northwest Women's and Children's Hospital, Xi'an, China.

\section{Patients and groups}

This study included 4,114 cycles of infertile patients who underwent IVF or ICSI treatment between January 2014 and May 2017. All patients whose serum estradiol (E2) levels reached 4,800 pg/mL on the trigger day were divided into the combined- and single-treatment (control) groups. The inclusion criteria were couples with infertility, women undergoing IVF/ICSI treatments, E2 level of 4,800 pg/mL on trigger day, and those already undergoing prednisone and/or bromocriptine treatments. The exclusion criteria were cycles cancelled before oocyte retrieval, cycles with oocyte donation or sperm donation, abnormal liver or kidney function, and incomplete follow-up or medical records (Additional information 1).

\section{Ovarian stimulation and medications}

Patients with agonist protocol were monitored for the following: pituitary downregulation, maintenance of $\mathrm{E} 2<25 \mathrm{pg} / \mathrm{mL}$, maintenance of luteinizing hormone $(\mathrm{LH})<5 \mathrm{U} / \mathrm{L}$, a diameter of the ovarian follicles $<$ $5 \mathrm{~mm}$ (monitored by B-ultrasound), and endometrial thickness $<5 \mathrm{~mm}$. Patients who met the inclusion criteria underwent ovarian stimulation with an initial gonadotropin dose of 150-225 IU daily, which was adjusted according to the patients' body mass index (BMI), ovarian antral follicle count (AFC), ovarian reserve, and ovarian response in previous IVF/ICSI cycles. Follicle development and E2 levels were monitored by transvaginal sonography and blood testing. The dose adjustments were based on hormonal level changes, ovarian volume, oocyte sizes, and numbers. When at least two dominant follicles reached $18 \mathrm{~mm}$ in diameter or three follicles reached $17 \mathrm{~mm}$ in diameter, $250 \mu \mathrm{g}$ recombinant human chorionic gonadotropin was administered as a trigger. Patients in the combined-treatment group received tablet bromocriptine $(2.5 \mathrm{mg})$ for rectal insertion and prednisone $(5 \mathrm{mg})$ for oral administration. The patients in the single-treatment group only received bromocriptine $2.5 \mathrm{mg}$ for rectal insertion. These two groups received their respective treatment from the trigger day up to 7 days after oocyte retrieval.

\section{Statistical analysis}

Statistical analysis was performed using IBM SPSS Statistics for Windows version 23.0 (IBM Corp., Armonk, NY, USA). Results are presented as mean \pm standard deviation (SD) or as a percentage (\%). Independent sample $t$-test was used for quantitative data with normal distribution, whereas the Wilcoxon test was used for quantitative data with non-normal distribution. The measured data were compared with analysis of variance. A chi-squared $\left(\chi^{2}\right)$ test was used for the classification data. $P<0.05$ was considered statistically significant.

\section{Results}

\section{Basic characteristics}


The basic characteristics of the patients in the combined- and single-treatment groups are presented in Table 1. The infertility factors were male factors ( 465 cycles), tubal factors ( 848 cycles), anovulation (125 cycles), endometriosis ( 17 cycles), and bilateral factors of the couple (289 cycles) in the combinedtreatment group and male factors ( 452 cycles), tubal factors (859 cycles), anovulation ( 172 cycles), endometriosis ( 14 cycles), and bilateral factors of the couple (315 cycles) in the single-treatment group. All infertile patients underwent controlled ovarian hyperstimulation. The causes of infertility, such as anovulatory factor, fallopian tube factor, endometriosis, male factor, and other factors, were compared, and the fallopian tube factor was determined to be the most common infertility marker between the two groups (Additional information 2). There were statistical differences in endometrial thickness on trigger day, total gonadotropin dose and duration, hormone levels on trigger day, and the number of actual oocyte retrievals; meanwhile, changes in hormone levels were statistically different in some, but not in others (Additional information 3).

Table 1

Basic characteristics of the study population

\begin{tabular}{|c|c|c|c|}
\hline Characteristics & $\begin{array}{l}\text { Combined-treatment group }(n= \\
2,057)\end{array}$ & $\begin{array}{l}\text { Single-treatment group }(n= \\
2,057)\end{array}$ & $P$ \\
\hline Age (years) & $29.30 \pm 3.79$ & $29.06 \pm 3.89$ & 0.055 \\
\hline Cycles & $1.12 \pm 0.408$ & $1.10 \pm 0.395$ & 0.119 \\
\hline $\begin{array}{l}\text { Basal FSH } \\
(\mathrm{mlU} / \mathrm{mL})\end{array}$ & $6.34 \pm 1.74$ & $6.25 \pm 1.74$ & 0.071 \\
\hline $\begin{array}{l}\text { Basal LH } \\
(\mathrm{mlU} / \mathrm{mL})\end{array}$ & $5.93 \pm 4.45$ & $6.10 \pm 3.78$ & 0.179 \\
\hline Basal E2 (pg/mL) & $40.64 \pm 18.58$ & $41.29 \pm 17.66$ & 0.263 \\
\hline Basal P (ng/mL) & $0.65 \pm 0.42$ & $0.62 \pm 0.60$ & 0.054 \\
\hline $\mathrm{AMH}(\mathrm{ng} / \mathrm{mL})$ & $5.19 \pm 4.32$ & $6.38 \pm 3.85$ & 0.059 \\
\hline AFC & $7.72 \pm 2.72$ & $7.89 \pm 2.79$ & 0.060 \\
\hline $\mathrm{P} / \mathrm{S}$ & $1237 / 807$ & $1277 / 776$ & 0.269 \\
\hline $\begin{array}{l}\text { Duration of } \\
\text { infertility }\end{array}$ & $3.45 \pm 2.319$ & $3.52 \pm 2.416$ & 0.287 \\
\hline BMI $\left(\mathrm{kg} / \mathrm{m}^{2}\right)$ & $21.65 \pm 3.08$ & $22.02 \pm 8.56$ & 0.067 \\
\hline \multicolumn{4}{|c|}{ Values are presented as mean \pm standard deviation. } \\
\hline \multicolumn{4}{|c|}{$P<0.05$, statistically significant. } \\
\hline \multicolumn{4}{|c|}{$\begin{array}{l}\text { FSH, follicle-stimulating hormone; LH, luteinizing hormone; E2, estradiol; P, progesterone; } A M H \text {, anti- } \\
\text { Müllerian hormone; AFC, antral follicle counts; P/S, primary infertility/secondary infertility; BMI, body } \\
\text { mass index. }\end{array}$} \\
\hline
\end{tabular}




\section{Fresh transfer cycles cancellation for OHSS}

Some cycles were cancelled due to the occurrence of symptoms of OHSS and other reasons such as hydrosalpinx, abnormally high progesterone levels, endometriosis, preimplantation genetic diagnosis, preimplantation genetic screening, intrauterine effusion or abnormal endometrium, or patients' personal reasons. The OHSS cancellation rate in the single-treatment group was significantly higher than that of the combined-treatment group (Table 2).

Table 2

Cancellation of the fresh transfer cycle in the combined- and single-treatment groups

\begin{tabular}{|c|c|c|c|c|}
\hline $\begin{array}{l}\text { Reasons for } \\
\text { cancellation }\end{array}$ & $\begin{array}{l}\text { Combined-treatment group } \\
(n=2,057)\end{array}$ & $\begin{array}{l}\text { Single-treatment group ( } n \\
=2,057 \text { ) }\end{array}$ & $\chi^{2}$ & $P$ \\
\hline OHSS $(n, \%)$ & $648(31.50)$ & $841(40.88)$ & 36.625 & $<0.001$ \\
\hline Others (n, \%) & $563(27.6)$ & $463(22.5)$ & & \\
\hline \multicolumn{5}{|c|}{ Values are presented as $\mathrm{n}$ (cancellation rate, \%). } \\
\hline
\end{tabular}

\section{Embryo culture and transfer}

A total of 1,601 cycles for fresh transfer were included in this study, whereas the other 2,513 cycles were cancelled. Of all the fresh cycles, 846 cycles were in the combined-treatment group, which included 826 cycles of the agonist protocol and 20 cycles of the antagonist protocol. Meanwhile, 753 cycles were in the single-treatment group, including 727 cycles with the agonist protocol and 26 cycles with the antagonist protocol. There was no statistical difference in the proportion of the different protocols for the fresh transfer cycles between the two groups (Additional information 4).

We compared the embryo culture and transfer between the two groups of fresh transfer cycles (Table 3). The embryos were defined by embryologists according to the embryo evaluation standard [27], with grade 1 and 2 embryos considered to be high-quality embryos, and the patients' age, heights, cesarean delivery history, as well as other surgical histories, were used to calculate the optimal embryo numbers. In the combined-treatment group, the number of high-quality embryos was significantly higher than that in single-treatment group. The average number of embryo transfer in the single-treatment group was higher than the combined-treatment group (Table 3). 
Table 3

The embryo culture and transfer in the fresh cycle

\begin{tabular}{|llll|}
\hline & Combined & Single & $P$ \\
\hline High-quality embryos numbers & 3,670 & 3,105 & $t=1.463, p=0.144$ \\
\hline Average embryo transfer number & 1.59 & 1.70 & \\
\hline Transfer types & & & \\
\hline Single transfer & 350 & 227 & $\chi 2=20.361, p<0.001^{\star *}$ \\
\hline Double transfer & 495 & 517 & \\
\hline Total transfer cycles & 846 & 753 & $t=-2.527, p=0.012^{*}$ \\
\hline Implantation numbers (IR) & $509(40.88 \%)$ & $442(38.30 \%)$ & $t=0.082, p=0.935$ \\
\hline Blastocysts numbers (BFR) & $2,535(51.95 \%)$ & $2,248(51.76 \%)$ & \\
\hline Note: ${ }^{*} P<0.05$ and $* \star P<0.001$, statistically significant. & & \\
\hline$P<0.05$, statistically significant. & & \\
\hline
\end{tabular}

\section{Clinical pregnancy outcomes}

There was no statistical difference in clinical pregnancy, ongoing pregnancy, and live birth rates. Early abortion occurred in 50 cycles in the combined-treatment group, but 47 cycles in the single-treatment group showed no statistical difference $(P=0.792)$. Fetal malformation occurred in five cases in the combined-treatment group and three cases in the single-treatment group $(P=0.081)$. Ectopic pregnancy occurred in six cases in the combined-treatment group and in four cases in the single-treatment group, which was not statistically different $(P=0.649)$. There was no statistically significant difference in the biochemical pregnancy rate and congenital abnormalities (Table 4). Although there was a significant difference in the OHSS cancellation rate between the two groups, there were no statistical differences in pregnancy outcomes. 
Table 4

Embryo transfer outcomes in the fresh transfer cycle of the two groups.

\begin{tabular}{|lllll|}
\hline & $\begin{array}{l}\text { Combined-treatment } \\
\text { group }\end{array}$ & $\begin{array}{l}\text { Single-treatment } \\
\text { group }\end{array}$ & X2 & P \\
\hline Clinical pregnancy rate & $60.52(512)$ & $58.83(443)$ & 0.472 & 0.492 \\
\hline Biochemical pregnancy rate & $7.23(37)$ & $7.90(35)$ & 0.070 & 0.792 \\
\hline Ongoing pregnancy rate & $54.02(457)$ & $53.12(400)$ & 0.129 & 0.719 \\
\hline $\begin{array}{l}\text { Ectopic pregnancy occurrence } \\
\text { rate }\end{array}$ & $1.17(6)$ & $0.90(4)$ & 0.207 & 0.649 \\
\hline Miscarriage rate & $9.77(50)$ & $10.61(47)$ & 0.070 & 0.792 \\
\hline Fetal malformation rate & $0.98(5)$ & $0.68(3)$ & 3.050 & 0.081 \\
\hline Live birth rate & $53.31(451)$ & $51.13(385)$ & 1.097 & 0.295 \\
\hline Birth defects rate & $0.67(4)$ & $1.04(4)$ & 0.349 & 0.554 \\
\hline Values are presented as \% $(\mathrm{n})$. $\mathrm{n}$ : cycles number & & & \\
\hline$P<0.05$, statistically significant. & & & & \\
\hline
\end{tabular}

\section{Discussion}

OHSS is a series of symptoms caused by excessive ovarian responses to exogenous gonadotropins. Its specific pathogenesis is unclear and possibly includes increased capillary permeability, decreased perfusion of essential organs, electrolyte disturbance, and blood concentration [28]. The clinical manifestations are bilateral ovarian enlargement, pleural ascites, abdominal distension, liver and kidney dysfunctions, and other serious complications. VEGF, due to its angiogenic properties, is currently considered a critical factor for the occurrence of OHSS [29]. The risk factors for OHSS include age $<35$ years, polycystic ovary syndrome or polycystic ovarian changes, $\mathrm{BMI}<18.5$ or $>24$, anti-Müllerian hormone $(A M H)>3.36 \mathrm{ng} / \mathrm{mL}$, increased number of sinus follicles (increased AFC is also a sensitive predictor for OHSS), increased follicular development and oocyte retrieval numbers, and OHSS history in the former cycles.

A study showed that the OHSS risks for young infertile patients aged $<35$ years were higher than those for patients aged $>35$ years [30]. Danninger et al. [31] followed 101 patients with IVF-assisted pregnancy and found that patients with OHSS had a significantly lower BMI than those without OHSS. AMH can identify ovarian reactivity to exogenous gonadotropins during the ovulation cycle. Lee et al. [32] found that AMH was a predictor of OHSS when it was higher than $3.36 \mathrm{ng} / \mathrm{mL}$. Jayaprakasan et al. [33] and other researchers [34] have shown that the greater the number of AFCs for infertile patients, the higher the incidence and risks of moderate to severe OHSS. Thus, this retrospective study evaluated if combined treatment with prednisone and bromocriptine is better than single-treatment with bromocriptine for OHSS 
prevention and whether it will influence pregnancy outcomes. Some differences in gonadotropin duration and endometrial thickness on trigger day were found between the two groups (Table 2). Although the gonadotropin duration was different, which led to different progesterone and LH levels on the trigger day, the gonadotropin doses and E2 levels were not statistically different between the two groups. Based on the OHSS diagnostic standard, some patients in this study only had symptoms of mild OHSS in some cycles, while a few others experienced no symptoms of abdominal distension, discomfort, mild nausea/vomiting, or diarrhea and only showed an increased E2 level.

The significant difference in the OHSS cancellation rate $31.50 \%$ in the combined-treatment group and $40.88 \%$ in the single-treatment group) indicated that combined treatment with prednisone and bromocriptine was better than bromocriptine alone for relieving OHSS and also for dramatically decreasing the cancellation rate for the fresh embryo transfer cycle. Also, there was no statistical difference in the proportion of the controlled ovarian stimulation protocol between these two groups, which mainly existed in the agonist protocols, but the antagonist protocols were in the minority. Analysis of embryo quality and embryo transfer outcome indicated that the combination of bromocriptine and prednisone can relieve the symptoms of OHSS but would not influence the quality of the transferred embryos.

Regarding pregnancy outcomes, there were no statistical differences in clinical pregnancy, ongoing pregnancy, live birth, and early miscarriage rates between the two groups. Although the cycles of the single-treatment group to which only one embryo was transferred were fewer than those of the combinedtreatment group, the implantation rate in the combined-treatment group was significantly better than that in the single-treatment group. This demonstrated that the advantages of selective embryo transfer and the pregnancy outcome may be closely related to the embryo quality itself and have little to do with the number of embryo transfers. Although fetal malformation occurred in five cycles in the combinedtreatment group and three cycles in the single-treatment group (including congenital heart disease, sixfinger deformity, and abdominal fissure malformation), there was no significant difference in the incidence of fetal malformation and ectopic pregnancy between the two groups. Furthermore, there were no significant statistical differences in the rates of biochemical pregnancy and congenital disabilities between the combined- and single-treatment groups. This led us to believe that when used in combination as an intervention for susceptible patients (that lead to OHSS during the controlled ovarian stimulation medication), both had no influence the pregnancy outcome even for the malformation or some congenital disease and also showed no side effects.

This study has a few limitations. Since this is a retrospective study, all relevant data were not randomized. Therefore, randomized experimental research will have to be designed to obtain more reliable results to confirm whether prednisone combined with bromocriptine could have a better effect on OHSS prevention in fresh transfer cycles. We should also design a new group study for patients with a high responder and for a normal responder to make sure if these combined treatments would have different effects on diverse groups of patients. 


\section{Conclusion}

Compared with bromocriptine alone, combined treatment with bromocriptine and prednisone can better reduce the OHSS occurrence rate and does not affect the pregnancy outcomes in fresh embryo transfer cycles. This can be beneficial for patients wanting to perform a fresh transfer and also for those with higher E2 levels during the whole ovarian stimulation.

\section{Abbreviations}

AFC: antral follicle counts

AMH: anti-Müllerian hormone

ART: assisted reproduction technology

BMl: body mass index

E2: estradiol

FSH: follicle-stimulating hormone

ICSI: intracytoplasmic sperm injection

IVF: in vitro fertilization

LH: luteinizing hormone

OHSS: ovarian hyperstimulation syndrome

P/S: primary infertility/secondary infertility

VEGF: vascular endothelial growth fact

\section{Declarations}

\section{Ethics approval and consent to participate:}

This study was approved by the Ethics Review Board of Northwest Women's and Children's Hospital, Xi'an, China. When we use the data that come from the patients, we all have been received their conformed consents.

\section{Consent for publication:}

Not applicable

Availability of data and materials: 
The datasets used and analyzed during the current study are available from the corresponding author on reasonable request.

\section{Competing interests:}

The authors declare that they have no competing interests.

\section{Funding:}

Not applicable

\section{Authors' contributions:}

TW collected, sorted, and analyzed the data. YX performed a major contribution to the statistical analysis of data and writing of the manuscript. Both authors contributed equally to this work and have read and approved the final manuscript.

\section{Acknowledgements:}

We thank Bing LIAN and Dan-huan LIN for their helps in this study.

\section{References}

1. Prevention and treatment of moderate and severe ovarian hyperstimulation syndrome: a guideline.Fertil Steril.2016;106:1634-47.

2. Aboulghar M.Prediction of ovarian hyperstimulation syndrome (OHSS). Estradiol level has an important role in the prediction of OHSS.Hum Reprod.2003;18:1140-1.

3. Delvigne A, Rozenberg S.Review of clinical course and treatment of ovarian hyperstimulation syndrome (OHSS).Hum Reprod Update.2003;9:77-96.

4. Nastri CO, Teixeira DM, Moroni RM, Leitão VM, Martins WP.Ovarian hyperstimulation syndrome: pathophysiology, staging, prediction and prevention.Ultrasound Obstet Gynecol.2015;45:377-93.

5. Sherwal V, Malik S, Bhatia V.Effect of bromocriptine on the severity of ovarian hyperstimulation syndrome and outcome in high responders undergoing assisted reproduction.J Hum Reprod Sci.2010;3:85-90.

6. Fan J, Zhong Y, Chen C.Combined treatment of prednisone and aspirin, starting before ovulation induction, may improve reproductive outcomes in ANA-positive patients.Am J Reprod Immunol.2016;76:391-95.

7. Nyborg KM, Kolte AM, Larsen EC, Christiansen OB.Immunomodulatory treatment with intravenous immunoglobulin and prednisone in patients with recurrent miscarriage and implantation failure after in vitro fertilization/intracytoplasmic sperm injection.Fertil Steril.2014;102:1650-55.

8. Zhu Q, Wu L, Xu B, Hu MH, Tong XH, Ji JJ, Liu YS.A retrospective study on IVF/ICSI outcome in patients with anti-nuclear antibodies: the effects of prednisone plus low-dose aspirin adjuvant 
treatment.Reprod Biol Endocrinol.2013;11:98.

9. Lu Y, Yan J, Liu J, Tan J, Hong Y, Wei D, Chen ZJ, Sun Y.Prednisone for patients with recurrent implantation failure: study protocol for a double-blind, multicenter, randomized, placebo-controlled trial.Trials.2020;21:719.

10. Robertson SA, Jin M, Yu D, Moldenhauer LM, Davies MJ, Hull ML, Norman RJ.Corticosteroid therapy in assisted reproduction - immune suppression is a faulty premise.Hum Reprod.2016;31:2164-73.

11. Tan SL, Balen A, el Hussein E, Campbell S, Jacobs HS. The administration of glucocorticoids for the prevention of ovarian hyperstimulation syndrome in in vitro fertilization: a prospective randomized study.Fertil Steril.1992;58:378-83.

12. Lainas T, Petsas G, Stavropoulou G, Alexopoulou E, Iliadis G, Minaretzis D.Administration of methylprednisolone to prevent severe ovarian hyperstimulation syndrome in patients undergoing in vitro fertilization.Fertil Steril.2002;78:529-33.

13. Mottla GL, Smotrich DB, Gindoff PR, Stillman RJ.Increasing clinical pregnancy rates after IVF/ET. Can immunosuppression help?J Reprod Med.1996;41:889-91.

14. Han AR, Ahn H, Vu P, Park JC, Gilman-Sachs A, Beaman K, Kwak-Kim J. Obstetrical outcome of antiinflammatory and anticoagulation therapy in women with recurrent pregnancy loss or unexplained infertility.Am J Reprod Immunol.2012;68:418-27.

15. Naredi N, Talwar P, Sandeep K. VEGF antagonist for the prevention of ovarian hyperstimulation syndrome: Current status.Med J Armed Forces India.2014;70:58-63.

16. Tang H, Mourad S, Zhai SD, Hart RJ.Dopamine agonists for preventing ovarian hyperstimulation syndrome.Cochrane Database Syst Rev.2016;11:Cd008605.

17. Alvarez C, Martí-Bonmatí L, Novella-Maestre E, Sanz R, Gómez R, Fernández-Sánchez M, Simón C, Pellicer A.Dopamine agonist cabergoline reduces hemoconcentration and ascites in hyperstimulated women undergoing assisted reproduction.J Clin Endocrinol Metab.2007;92:2931-7.

18. Chen SU, Chou CH, Lin CW, Lee H, Wu JC, Lu HF, Chen CD, Yang YS.Signal mechanisms of vascular endothelial growth factor and interleukin-8 in ovarian hyperstimulation syndrome: dopamine targets their common pathways.Hum Reprod.2010;25:757-67.

19. Garcia-Velasco JA. How to avoid ovarian hyperstimulation syndrome: a new indication for dopamine agonists.Reprod Biomed Online.2009;18 Suppl 2:71-5.

20. Shaltout A, Shohyab A, Youssef MA.Can dopamine agonist at a low dose reduce ovarian hyperstimulation syndrome in women at risk undergoing ICSI treatment cycles? A randomized controlled study.Eur J Obstet Gynecol Reprod Biol.2012;165:254-8.

21. Shrem G, Steiner N, Balayla J, Volodarsky-Perel A, Tannus S, Son WY, Dahan MH.Use of cabergoline and post-collection GnRH antagonist administration for prevention of ovarian hyperstimulation syndrome.Reprod Biomed Online.2019;39:433-38.

22. Bassiouny YA, Dakhly DMR, Bayoumi YA, Salaheldin NM, Gouda HM, Hassan AA. Randomized trial of combined cabergoline and coasting in preventing ovarian hyperstimulation syndrome during in vitro fertilization/intracytoplasmic sperm injection cycles. 
23. Spitzer D, Wogatzky J, Murtinger M, Zech MH, Haidbauer R, Zech NH.Dopamine agonist bromocriptine for the prevention of ovarian hyperstimulation syndrome.Fertil Steril.2011;95:27424.e1.

24. Youssef MA, van Wely M, Hassan MA, Al-Inany HG, Mochtar M, Khattab S, van der Veen F.Can dopamine agonists reduce the incidence and severity of OHSS in IVF/ICSI treatment cycles? A systematic review and meta-analysis.Hum Reprod Update.2010;16:459-66.

25. Kasum M, Vrcic H, Stanic P, Jezek D, Oreskovic S, Beketic-Oreskovic L, Pekez M.Dopamine agonists in prevention of ovarian hyperstimulation syndrome.Gynecol Endocrinol.2014;30:845-9.

26. Mourad S, Brown J, Farquhar C.Interventions for the prevention of OHSS in ART cycles: an overview of Cochrane reviews.Cochrane Database Syst Rev.2017;1:Cd012103.

27. Pantos K, Makrakis E, Chronopoulou M, Biba M, Perdikaris A, Dafereras A.Day 4 versus day 3 embryo transfer: a prospective study of clinical outcomes.Fertil Steril.2008;89:573-7.

28. Beerendonk CC, van Dop PA, Braat DD, Merkus JM. Ovarian hyperstimulation syndrome: facts and fallacies.Obstet Gynecol Surv.1998;53:439-49.

29. Soares SR, Gómez R, Simón C, García-Velasco JA, Pellicer A. Targeting the vascular endothelial growth factor system to prevent ovarian hyperstimulation syndrome.Hum Reprod Update.2008;14:321-33.

30. Klemetti R, Sevón T, Gissler M, Hemminki E.Complications of IVF and ovulation induction.Hum Reprod.2005;20:3293-300.

31. Danninger B, Brunner M, Obruca A, Feichtinger W.Prediction of ovarian hyperstimulation syndrome by ultrasound volumetric assessment [corrected] of baseline ovarian volume prior to stimulation. Hum Reprod.1996;11:1597-9.

32. Lee TH, Liu CH, Huang CC, Wu YL, Shih YT, Ho HN, Yang YS, Lee MS.Serum anti-Müllerian hormone and estradiol levels as predictors of ovarian hyperstimulation syndrome in assisted reproduction technology cycles.Hum Reprod.2008;23:160-7.

33. Jayaprakasan K, Chan Y, Islam R, Haoula Z, Hopkisson J, Coomarasamy A, Raine-Fenning N.Prediction of in vitro fertilization outcome at different antral follicle count thresholds in a prospective cohort of 1,012 women.Fertil Steril.2012;98:657-63.

34. Zheng M, Zuo G, Tong J, Chen ZJ, Li WP, Zhang C.Intrafollicular melatonin concentration is elevated in patients with ovarian hyperstimulation syndrome (OHSS) and can serve as an important predictor of OHSS.Arch Gynecol Obstet.2019;299:1151-58.

\section{Supplementary Files}

This is a list of supplementary files associated with this preprint. Click to download.

- supplementfigure1.docx

- SupplementFigure2.docx 
- supplementtables.docx

Page 14/14 